\title{
Étude par spectroscopie de résonance paramagnétique électronique de la photodégradation des lignines extraites du bois de pin radiata (Pinus radiata D. Don)
}

\author{
Cyril Kamoun ${ }^{\mathrm{a}}$, André Merlin ${ }^{\mathrm{a}}$, Xavier Deglise ${ }^{\mathrm{a}}$, Silvio H. Urizar ${ }^{\mathrm{b}}$, Anna Maria Fernandez \\ a Laboratoire d'étude et de recherche sur le matériau bois : équipe photochimie appliquée et physicochimie des polymères, \\ université Henri Poincaré Nancy 1/Enstib BP 239, 54506 Vandœuvre les Nancy cedex, France \\ b Universidad del Bio-Bio, Casilla 5-C, Concepcion, Chili
}

(Reçu le 17 août 1998 ; accepté le 2 février 1999)

\begin{abstract}
ESR study of photodegradation of lignins extracted and isolated from Pinus radiata wood. Cellulose in lignocellulosic materials is protected from photochemical degradation by the lignin which can play a three-fold role: to filter near UV radiation by absorbing photons, to deactivate the excited state of cellulose, and by acting as an antioxidant by forming stable phenoxy radicals by radical transfer reactions. In the work presented we have studied by electron paramagnetic resonance (EPR) the photodegradation of lignins extracted and isolated from Pinus radiata wood. The kinetics of the intermediate radical species concentrations have been followed during irradiation of solid specimens as well as for lignins in solution both for the raw and for modified extracts after stopping light irradiation and stocking the samples in the dark in order to quantify the stability of the formed radicals. Attribution of EPR signals to phenoxy radicals formed by abstraction of an hydrogen atom from the phenolic chromophore groups of lignin has been confirmed by the study of radical transfer reactions from the $\mathrm{DPPH}^{\circ}$ radical to the lignins. The lignin ability to form resonance stabilized phenoxyl radicals confers on these materials antioxidant properties which allow the value-added use of this by-product of the pulp and paper industry. (C) 1999 Éditions scientifiques et médicales Elsevier SAS.
\end{abstract}

lignins / radiata pine / antioxidant / photodegradation / EPR spectroscopy

Résumé - Dans les matériaux lignocellulosiques, la cellulose est protégée de la dégradation photochimique par les lignines qui peuvent jouer un triple rôle : filtre en absorbant les photons du proche ultraviolet, sensibilisateur en désactivant les états excités de la cellulose, antioxydant en formant des radicaux phénoxyles par des réactions de transfert radicalaire. Dans ce travail, nous avons étudié par spectroscopie de résonance paramagnétique électronique (RPE) la photodégradation de lignines isolées extraites du bois de pin radiata. Les évolutions cinétiques de la concentration des espèces radicalaires intermédiaires ont été suivies, d'une part lors de l'irradiation des échantillons solides ou en solution de lignines soit brutes d'extraction soit modifiées chimiquement, d'autre part après arrêt de l'excitation lumineuse et stockage à l'obscurité pour quantifier la stabilité des radicaux formés. L'attribution du signal RPE à des radicaux phénoxyles formés par arrachement d'un atome d'hydrogène des chromophores phénoliques des lignines a été confirmée par une étude de la réaction de transfert radicalaire du radical diphénylpicrylhydrazyle (DPPH ${ }^{\circ}$ ) sur les lignines. Cette faculté des lignines à former des radicaux phénoxyles stabilisés par résonance leur confère des propriétés antioxydantes qui peuvent permettre une valorisation de ce sous produit de l'industrie papetière. (C) 1999 Éditions scientifiques et médicales Elsevier SAS.

lignines / pin radiata / antioxydant, photodégradation / spectroscopie RPE

\footnotetext{
* Correspondence and reprints merlin@lermab.u-nancy.fr
} 


\section{Introduction}

Par des chromophores phénoliques présents dans leur structure moléculaire, les lignines représentent le constituant principal du bois le plus affecté par le rayonnement solaire. Les altérations des propriétés de surface du bois soumis à une lumière de type solaire sont principalement imputables à ce composé et il a été montré que les photoproduits stables responsables des modifications de la couleur du bois ont pour origine exclusivement des espèces radicalaires formées par la photolyse des lignines $[11,12,36]$. Une connaissance, à l'échelle moléculaire, des réactions induites par l'irradiation des lignines est nécessaire d'une part pour comprendre les mécanismes d'altération à la lumière des propriétés de surface du bois et d'autre part pour proposer des solutions adaptées pour améliorer la durabilité de l'aspect naturel de ce matériau. La dégradation photochimique des lignines étant de nature radicalaire, la spectroscopie de résonance paramagnétique électronique apparaît la technique la mieux adaptée à une étude de cinétique de la photodégradation de ce constituant du bois.

Le bois de pin radiata (Pinus radiata D. Don ou Pinus insignis Dougl.) est largement utilisé en menuiserie extérieure au Chili, de telle sorte que l'augmentation de sa durabilité représente un véritable enjeu économique pour ce pays.

Dans ce travail, nous nous sommes attachés à dégager le rôle des chromophores phénoliques des lignines en suivant, par spectroscopie de résonance paramagnétique électronique (RPE), les évolutions cinétiques de la concentration en espèces radicalaires intermédiaires :

- lors de l'irradiation d'échantillons solides ou en solution de lignines de cette essence soit brutes d'extraction soit modifiées chimiquement ;

- après arrêt de l'excitation lumineuse et stockage à l'obscurité pour quantifier la stabilité de ces radicaux libres.

La spectroscopie de résonance paramagnétique électronique constitue une méthode directe de mesure du paramagnétisme des radicaux libres très sensible. Cette technique est particulièrement bien adaptée aux études cinétiques de formation des radicaux lors des réactions de photodégradation des matériaux lignocellulosiques [23].

\section{Matériel et méthodes}

La lignine brute a été extraite du bois de pin radiata par un mélange acide formique acétone (70/30) suivant un protocole préétabli $[51,52]$. Un traitement à l'acide acétique en solution diluée ( $35 \%$ ) de cette lignine brute permet une élimination des hydrates de carbone (lignine purifiée). Nous avons également étudié une lignine modifiée chimiquement obtenue à partir de la lignine purifiée par acétylation des groupes phénoliques (lignine acétylée).

Les évolutions des concentrations en radicaux libres ont été analysées à l'aide d'un spectromètre RPE Bruker type ER 200D fonctionnant en bande $X$ avec une cavité de mode TE 102 permettant une irradiation directe de l'échantillon en cours de mesure. Nous avons utilisé une lampe à arc au xénon (type Osram XBO $1000 \mathrm{~W}$ ) émettant un flux lumineux de l'ordre de $30 \mathrm{~mW} / \mathrm{cm}^{2}$ à $360 \mathrm{~nm}$ au niveau de l'échantillon. Le spectre d'émission dans le proche UV-visible de cette source lumineuse est comparable au spectre émis par le soleil de telle sorte que les lampes à arc au xenon sont souvent utilisées pour simuler la lumière solaire dans des études du vieillissement accéléré des matériaux. Les irradiations et les enregistrements des spectres RPE ont été conduits à température ambiante soit sur des échantillons de poudre de lignines (brute, purifiée et acétylée) soit sur des solutions dans le dioxane. Les spectres RPE du bois de pin radiata ont été enregistrés sur des petits bâtonnets de dimension $35 \times 3 \times 3 \mathrm{~mm}$. Afin d'obtenir des résultats reproductibles, nous nous sommes fixés certains paramètres expérimentaux comme la puissance de la microonde, la modulation de champ, la constante de temps... Pour dégager le rôle de la nature spectrale du rayonnement utilisé, les échantillons ont été analysés soit dans des tubes de quartz transparents à la totalité du rayonnement émis par la lampe soit dans des tubes de pyrex qui absorbent totalement les longueurs d'onde inférieures à 310 nm. Les positions des signaux RPE quantifiées par le facteur de Landé ont été calculées par comparaison au spectre du radical $\mathrm{DPPH}^{0}$ (diphénylpicrylhydrazyle) pour lequel le facteur de Landé $g$ est de 2,0023. Les courbes représentant les évolutions des concentrations en espèces radicalaires ont été modélisées par un programme informatique de lissage et les expressions analytiques des fonctions décrivant ces courbes ont été calculées en minimisant la somme des carrés des écarts entre la courbe calculée et le nuage des points expérimentaux.

\section{Résultats}

\subsection{Analyse des lignines en phase solide}

Un signal RPE est observé avant irradiation sur les échantillons de poudre des trois lignines étudiées, ce qui montre la présence de radicaux libres en absence d'excitation lumineuse. Pour ces trois types d'échantillons, nous avons mis en évidence un signal RPE de même 
forme et de même largeur : singulet d'environ 6,5 gauss de large avec un facteur de Landé de 2,0046. Les intensités de ces signaux RPE mesurées par la mi-hauteur de la courbe dérivée du signal d'absorption dépendent fortement de la nature de la lignine analysée :

\section{lignine purifiée $>$ lignine brute $>$ lignine acétylée} (rapport 14/7/1)

Ces résultats indiquent que, pour les trois types de lignines étudiées, on a, en absence de rayonnement, des radicaux libres de même nature mais en concentration très différente. Une analyse comparable de lignines extraites au dioxane du bois d'Abies grandis a montré que l'intensité de ce signal RPE est réduite à l'échelle du bruit de fond si nous prenons soin de stocker l'échantillon à l'obscurité avant la mesure [11]. Ces observations suggèrent la présence de radicaux libres stables dans les lignines dont la concentration augmente lors de la manipulation des échantillons à la lumière. Un signal RPE est également observé en absence d'excitation lumineuse sur le bois de pin radiata (figure 1). Le singulet central de ce spectre est analogue en largeur et en position au singulet observé lors de l'analyse des lignines. Il faut noter que l'intensité du signal RPE observé sur le bois de pin radiata est très faible (rapport d'intensité 1/100 par rapport à la lignine brute).

L'irradiation des poudres des trois lignines étudiées modifie uniquement l'intensité du signal RPE ; comme avant l'excitation lumineuse, on observe un signal singulet d'environ 7 gauss de large. L'irradiation ne modifie pas la nature des espèces radicalaires mais augmente leur concentration à l'état stationnaire.

De même, la nature spectrale du rayonnement n'a pas d'influence sur la nature des espèces radicalaires produites : les analyses des échantillons en tubes de quartz et de pyrex conduisent à des signaux RPE de mêmes caractéristiques. En revanche, les cinétiques sont affectées par la nature spectrale du rayonnement, en particulier les concentrations à l'état stationnaire sont plus importantes lors de l'irradiation avec la totalité du rayonnement émis par la lampe.

Dans nos conditions opératoires, il n'a pas été possible de détecter une éventuelle structure hyperfine des signaux RPE observés. En fixant le champ magnétique à la valeur correspondant au maximum de l'intensité du signal RPE, nous avons suivi l'évolution cinétique de l'intensité de ce signal avec le temps d'irradiation. Ces mesures ont été faites à l'air sur des lignines en poudre placées en tube de pyrex. Pour ces trois types de lignine, nous observons une évolution similaire :

- augmentation rapide de la concentration en radicaux en début d'irradiation ;

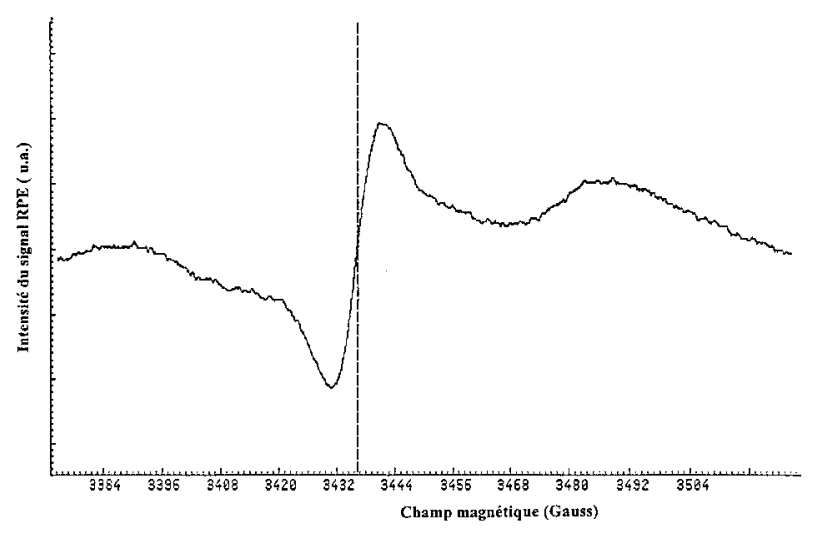

Figure 1. Spectre RPE du bois de pin radiata avant l'irradiation ; puissance microonde : $5 \mathrm{~mW}$; modulation : 4 Gpp ; fréquence microonde : 9,70 $\mathrm{GHz}$.

- stabilisation de la concentration en radicaux après environ 50 min d'exposition au rayonnement lumineux dans nos conditions opératoires.

Les variations de l'intensité du signal RPE en fonction de la durée d'irradiation sont correctement modélisées, pour les trois types d'échantillons, par une loi de la forme :

$$
\mathrm{I}(\mathrm{t})=\mathrm{I}(\mathrm{o})+[\mathrm{I}(\infty)-\mathrm{I}(\mathrm{o})][1-\exp (-\mathrm{t} / \tau)]
$$

(figure $2 a$ : exemple de la lignine purifiée) avec :

$\mathrm{I}(\mathrm{o})$ : intensité du signal avant irradiation

$\mathrm{I}(\infty)$ : intensité du signal à l'état stationnaire

$\tau$ : constante de temps qui permet de comparer les vitesses de formation des espèces radicalaires pour les différents types de lignines.

Cette loi qui peut s'écrire $\frac{I(\infty)-I(t)}{I(\infty)-I(0)}=\exp (-t \tau)$

correspond à une cinétique du premier ordre avec comme paramètre $\mathrm{I}(\infty)-\mathrm{I}(\mathrm{t})$. Les résultats obtenus par cette modélisation des mesures expérimentales sont reportés sur le tableau $I$.

Pour avoir des informations sur la stabilité des radicaux libres formés, nous avons suivi, dans les mêmes conditions de mesure que pour l'étude de la formation des radicaux, l'évolution de l'intensité du signal RPE après arrêt de l'excitation lumineuse.

Pour être sûr d'avoir atteint l'état stationnaire, la durée d'irradiation avant l'extinction a été fixée à $60 \mathrm{~min}$ 

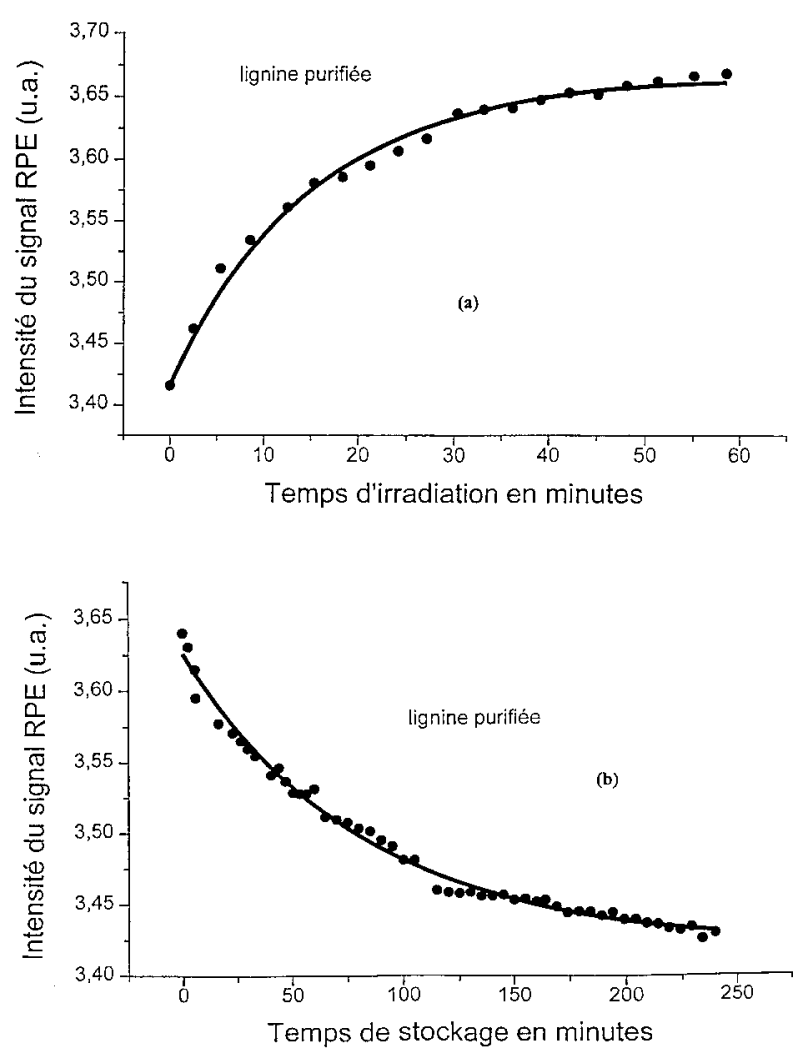

Figure 2. Modélisation des évolutions de l'intensité du signal RPE (cas de la lignine purifiée) : a) avec le temps d'irradiation ; b) avec le temps de stockage à l'obscurité. Les points correspondent aux mesures expérimentales, la courbe représente la modélisation. (figure 3). La décroissance de l'intensité du signal RPE a pu être modélisée pour les trois types de lignines étudiées, par une fonction d'expression analytique :

$$
I^{\prime}(t)=I^{\prime}(\infty)+\left[I^{\prime}(0)-I^{\prime}(\infty)\right] \exp \left(-t \tau^{\prime}\right)
$$

(figure $2 b$ : exemple de la lignine purifiée) avec:

$I^{\prime}(\mathrm{o})$ : intensité du signal à l'arrêt de l'excitation lumineuse

$I^{\prime}(\infty)$ : intensité du signal à l'état stationnaire

$\tau^{\prime}$ : constante de temps qui permet de comparer les vitesses de désactivation des radicaux donc d'estimer la stabilité de ces espèces.

Les résultats obtenus par le programme de modélisation sont reportés sur le tableau II.

Remarque : Ces modélisations des évolutions des intensités des signaux RPE ne rendent compte que de la globalité des réactions conduisant à la formation ou à la désactivation des espèces radicalaires. Il apparaît difficile d'interpréter de tels résultats à l'échelle moléculaire en terme d'ordre de réaction.

L'intensité $\mathrm{I}(\infty)$ du signal à l'état stationnaire est comparable à l'intensité du signal observé avant l'irradiation qui correspond à la concentration stationnaire en espèces radicalaires en lumière ambiante

Pour les trois types d'échantillons, les radicaux formés présentent une stabilité importante ; il faut environ $3 \mathrm{~h}$ pour revenir à la concentration initiale en radicaux avant l'excitation lumineuse (figure 3). L'ensemble des résultats que nous avons obtenu pour ces irradiations à l'air des échantillons de poudre montre que la purifica-

Tableau I. Modélisation des cinétiques de formation des espèces radicalaires.

\begin{tabular}{lccccc}
\hline Lignine : & $\mathrm{I}(0)$ u.a $;$ & $\mathrm{I}(\infty)$ u.a. & $\frac{\mathrm{I}(\infty)-\mathrm{I}(0)}{\mathrm{I}(0)}$ & $\begin{array}{c}\tau \text { en min } \\
\text { de corrélation }\end{array}$ \\
\hline purifiée & & 3,67 & $7 \%$ & 15,8 & 0,996 \\
brute & 3,43 & 1,81 & $9 \%$ & 7 & 0,989 \\
acétylée & 1,65 & 0,29 & $16 \%$ & 3 & 0,971 \\
\hline
\end{tabular}

Tableau II. Modélisation de la cinétique de desactivation des espèces radicalaires.

\begin{tabular}{|c|c|c|c|c|c|}
\hline Lignine : & $\mathrm{I}^{\prime}(\infty)$ u.a. & $I^{\prime}(0)$ u.a. & $\frac{I^{\prime}(0)-I^{\prime}(\infty)}{I^{\prime}(0)}$ & $\tau$ en min & $\begin{array}{l}\text { r coefficient } \\
\text { de corrélation }\end{array}$ \\
\hline purifiée & 3,42 & 3,67 & $6 \%$ & 41 & 0,993 \\
\hline acétylée & 0,24 & 0,28 & $16 \%$ & 38 & 0,980 \\
\hline
\end{tabular}




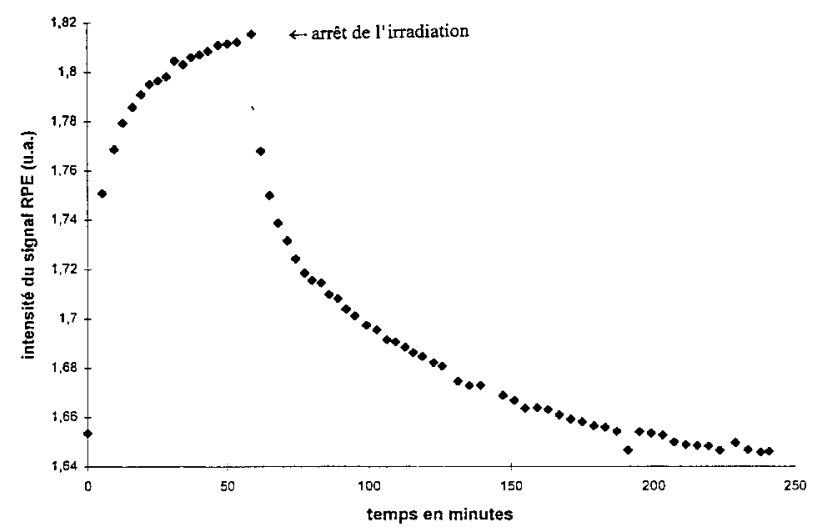

Figure 3. Évolution cinétique de l'intensité du signal RPE (cas de la lignine brute).

tion de la lignine brute ou l'acétylation de la lignine purifiée ne modifient pas la nature des radicaux formés ; on observe pour ces trois types d'échantillons analysés un signal RPE identique en forme et en position et des cinétiques de désactivation des espèces radicalaires comparables (tableau $I I$ ). En revanche les concentrations en radicaux avant irradiation et à l'état stationnaire lors de l'irradiation ainsi que les constantes de temps des réactions de formation (tableau I) sont fortement dépendantes de la nature des lignines avec pour ces trois paramètres :

lignine purifiée $>$ lignine brute $>$ lignine acétylée.

Il faut noter que les intensités des signaux RPE restent très faibles même après irradiation des échantillons en phase solide, ce qui entraîne une forte imprécision sur la détermination des paramètres cinétiques. Ce phénomène est accentué pour la lignine acétylée pour laquelle la détection du signal RPE est en limite des performances de notre appareillage. Les comportements différents observés entre les trois types d'échantillons peuvent être attribués à la concentration en chromophores de l'échantillon et donc à la différence d'énergie lumineuse absorbée. La purification de la lignine brute, en éliminant les hydrates de carbone qui n' absorbent pas le rayonnement de longueurs d'onde supérieures à $320 \mathrm{~nm}$, augmente de façon relative la concentration en chromophores de l'échantillon. L'acétylation par contre, modifie la nature chimique des chromophores des lignines et provoque une diminution de leur concentration.

Pour dégager le rôle de l'oxygène dans les processus de photodégradation de ces lignines, nous avons réalisé la même étude par spectroscopie RPE d'échantillons
Tableau III. Intensité du signal RPE avant irradiation.

\begin{tabular}{lcc}
\hline Lignine : & en présence d'oxygène & en absence d'oxygène \\
\hline brute & 1,66 & 0,79 \\
purifiée & 3,23 & 0,98 \\
acétylée & 0,25 & 0,26 \\
\hline
\end{tabular}

ayant subi un vide de l'ordre de $10^{-3}$ Torr pendant $2 \mathrm{~h}$. En absence d'oxygène, l'intensité du signal RPE avant irradiation est nettement affaiblie pour les lignines brutes et purifiées et ne semble pas affectée pour la lignine acétylée (tableau III). Nous avons observé sur un échantillon de lignine purifiée ayant subi le dégazage qu'une remise à l'atmosphère provoque une augmentation du signal RPE jusqu'à la valeur obtenue lors de l'analyse d'un échantillon non dégazé. Cette observation montre que la concentration stationnaire en radicaux avant l'irradiation est directement liée à la présence d'oxygène. Les intensités des signaux RPE augmentent très faiblement avec la durée d'irradiation pour les trois échantillons dégazés et il n'a pas été possible de proposer une analyse significative de la formation et de la désactivation des espèces radicalaires dans nos conditions expérimentales. Nous avons également mis en évidence qu'une remise à l'atmosphère d'un échantillon ayant subi le dégazage, soit au cours d'irradiation soit au cours du stockage à l'obscurité après arrêt de l'excitation lumineuse, provoque une augmentation immédiate du signal RPE. Ces résultats suggèrent, qu'en phase solide, la formation des espèces radicalaires est essentiellement liée à des phénomènes oxydatifs. Toutefois, même en absence d'oxygène, la formation des espèces radicalaires n'est pas totalement inhibée. La désactivation des espèces radicalaires est plus rapide en présence d'oxygène : sur un échantillon dégazé de lignine purifiée, nous avons observé que $12 \mathrm{~h}$ après arrêt de l'irradiation lumineuse, l'intensité du signal RPE est encore supérieure à celle mesurée avant l'irradiation.

\subsection{Analyse des lignines en solution dans le dioxane}

Les spectres d'absorption UV-visible des solutions dans le dioxane de même concentration massique $(40 \mathrm{mg}$ $\mathrm{L}^{-1}$ ) des trois types de lignines étudiées sont qualitativement d'allure comparable et présentent une structure continue peu résolue (figure 4). Ces spectres montrent que, pour les trois échantillons, les chromophores sont de même nature. 
Le calcul d'un coefficient d'extinction massique à 400 nm (tableau IV) montre que ces chromophores sont en concentration croissante selon la séquence :

lignine acétylée < lignine brute < lignine purifiée

Pour obtenir une concentration stationnaire en espèces radicalaires compatibles avec la mesure par spectroscopie RPE, nous avons dû irradier des solutions concentrées en lignines (de l'ordre de $20 \mathrm{~g} \mathrm{~L}^{-1}$ ). Dans ces conditions opératoires, d'une part l'absorption du rayonnement est totale et une analyse photochimique quantitative devient délicate ; d'autre part, la viscosité importante de ces solutions influence les cinétiques en limitant la diffusion des espèces. Comme pour l'analyse des poudres, nous obtenons pour les trois types d'échantillons un signal singulet d'environ 5,5 gauss de large avec un facteur de Landé de 2,0043. Cette observation met en évidence que les radicaux formés en solution sont de même nature qu'en phase solide et que les trois types de lignine produisent les mêmes espèces radicalaires. La figure 5 montre que les cinétiques de formation des espèces radicalaires sont plus complexes que pour la phase solide. En particulier, nous n'observons plus une concentration stationnaire. Les variations de l'intensité du signal RPE avec le temps d'irradiation sont correctement modélisées, pour les trois types de lignine, par une fonction dont l'expression analytique est de la forme :

$$
I(t)=K\left[\exp \left(-k_{1} t\right)-\exp \left(-k_{2} t\right)\right]+I(o) \exp \left(-k_{2} t\right)
$$

\section{(figure 5 et tableau $V$ )}

Cette expression analytique correspond à un schéma cinétique $A \underset{k_{i}}{\longrightarrow} B^{0} \underset{k_{2}}{\longrightarrow} C$ où seul le composé intermédiaire $\mathrm{B}^{0}$ est de nature radicalaire donc le seul détecté en spectroscopie RPE (cf. annexe 1).

Dans nos conditions opératoires, la concentration en radicaux au cours de l'irradiation serait globalement la résultante de deux réactions consécutives :

- une réaction du premier ordre (constante de temps $\tau_{1}=1 / \mathrm{k}_{1}$ ) de formation à partir des chromophores des lignines ;

- une réaction du premier ordre (constante de temps $\tau_{2}=1 / k_{2}$ ) de désactivation de ces espèces radicalaires.

Cette modélisation montre que les lignines brutes et purifiées présentent des contantes de temps $\tau_{1}$ et $\tau_{2}$ comparables (tableau $V$ ). Dans nos conditions opératoires, la vitesse de formation est faible devant la vitesse de désactivation des radicaux $\left(\mathrm{k}_{1}<<\mathrm{k}_{2}\right)$. Pour la lignine acétylée, la vitesse de formation est du même ordre de grandeur que pour les deux autres échantillons de lignine alors que la vitesse de désactivation est plus faible. $\mathrm{Ce}$
Tableau IV. Coefficient d'extinction massique à $400 \mathrm{~nm}$ des lignines étudiées

\begin{tabular}{lc}
\hline Lignine : & $\varepsilon$ à $400 \mathrm{~nm}$ en $\mathrm{L} . \mathrm{g}^{-1} \cdot \mathrm{cm}^{-1}$ \\
\hline acétylée & 5 \\
brute & 15 \\
purifiée & 31,5 \\
\hline
\end{tabular}

D.o.
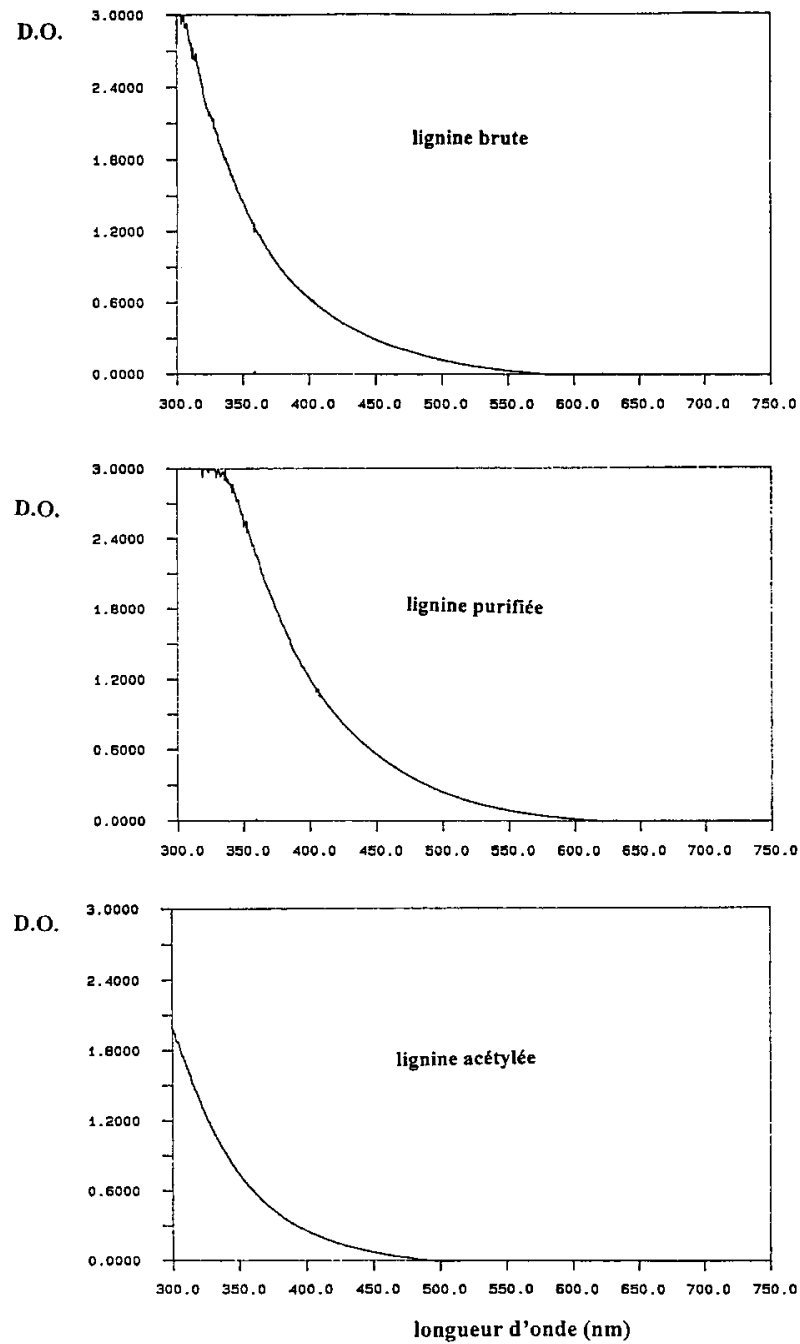

Figure 4. Spectres d'absorption UV-Visible des solutions de lignines $\left(40 \mathrm{mg} \mathrm{L}^{-1}\right)$ dans le dioxane.

résultat doit être pris avec précaution car pour la lignine acétylée, les intensités des signaux RPE sont en limite de détection de l'appareillage et les variations avec l'irradiation ne sont plus significatives. 

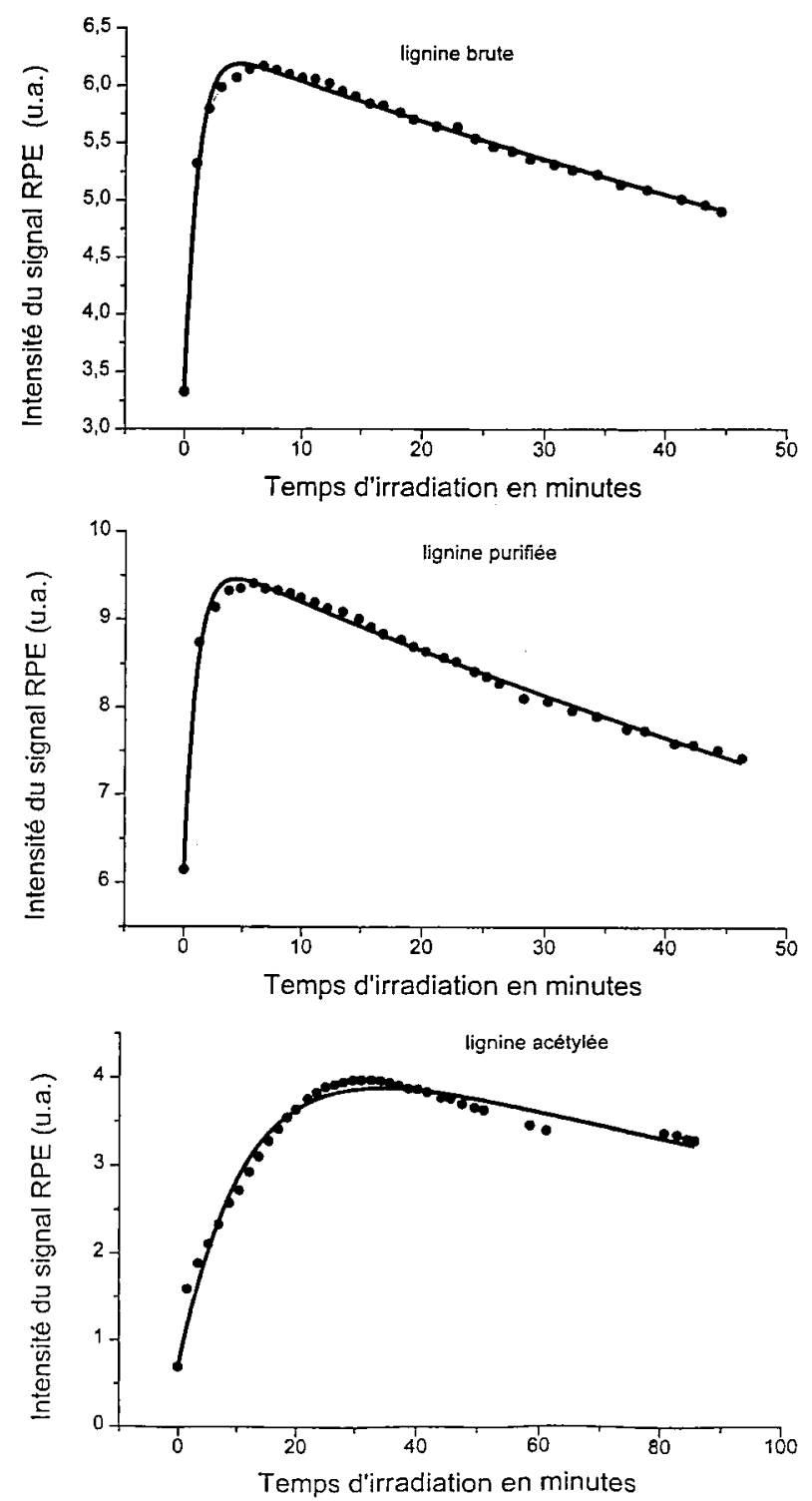

Figure 5. Modélisation des évolutions de l'intensité du signal RPE au cours de l'irradiation : lignines en solution dans le dioxane. Les points correspondent aux mesures expérimentales, la courbe représente la modélisation.
Le paramètre $K$ permet de comparer de façon relative, pour chaque type de lignine, les concentrations initiales de chromophores susceptibles de produire, après absorption de la lumière, les espèces radicalaires (annexe 1). Dans le tableau III, nous avons choisi la lignine brute comme référence. Ces résultats confirment les observations faites par spectroscopie d'absorption UV-visible tableau $I V)$ : l'acétylation provoque une diminution importante de la concentration initiale en chromophore alors que la purification de la lignine brute l'augmente.

Comme pour les analyses des lignines en poudre, la décroissance avec le temps de l'intensité du signal RPE après arrêt de l'excitation lumineuse a pu être correctement décrite pour les solutions de lignines brutes et purifiées par une expression de la forme :

$$
I^{\prime}(t)=I^{\prime}(\infty)+\left[I^{\prime}(0)-I(\infty)\right] \exp \left(-t / \tau^{\prime}\right)
$$

avec : I'(o) intensité du signal à l'arrêt de l'excitation lumineuse.

$I^{\prime}(\infty)$ : intensité du signal à l'état stationnaire.

Pour ces deux types de lignines, nous avons obtenu des constantes de temps très voisines (tableau III). Dans ce milieu, la cinétique de désactivation des espèces radicalaires est fortement accélérée par rapport à la phase solide. Pour la solution de lignine acétylée, les variations de l'intensité du signal RPE sont trop faibles pour obtenir une modélisation significative.

\section{Discussion}

Les études, à l'échelle moléculaire, des réactions de photodégradation des lignines ont été développées avec pour objectif d'expliquer les modifications des propriétés de surface du papier et du bois induites par une exposition à la lumière solaire. La structure des lignines extraites d'une essence résineuse proposée par Adler [1] met en évidence deux groupes chromophores (figure 6a) susceptibles d'intervenir dans la photodégradation solaire. Les spectres UV-visible de molécules modèles de ces deux chromophores $[2,53]$ présentent une absorption dans le domaine proche UV avec même pour le chromo-

Tableau V. Modélisation de la variation de l'intensité du signal RPE au cours de l'irradiation des lignines en solution dans le dioxane.

\begin{tabular}{lccccrr}
\hline Lignine : & $\mathrm{K}$ & $\mathrm{k}_{1}\left(\mathrm{~min}^{-1}\right)$ & $\tau_{1}(\min )$ & $\mathrm{k}_{2}\left(\mathrm{~min}^{-1}\right)$ & $\tau_{2}(\min )$ & $\begin{array}{c}\mathrm{r} \text { coefficient } \\
\text { de corrélation }\end{array}$ \\
\hline brute & 6,390 & 0,00594 & 168 & 0,9604 & 1,04 & 0,979 \\
purifiée & 9,766 & 0,00611 & 164 & 0,9584 & 1,04 & 0,991 \\
acétylée & 4,840 & 0,00479 & 208 & 0,0840 & 11,91 & 0,964 \\
\hline
\end{tabular}




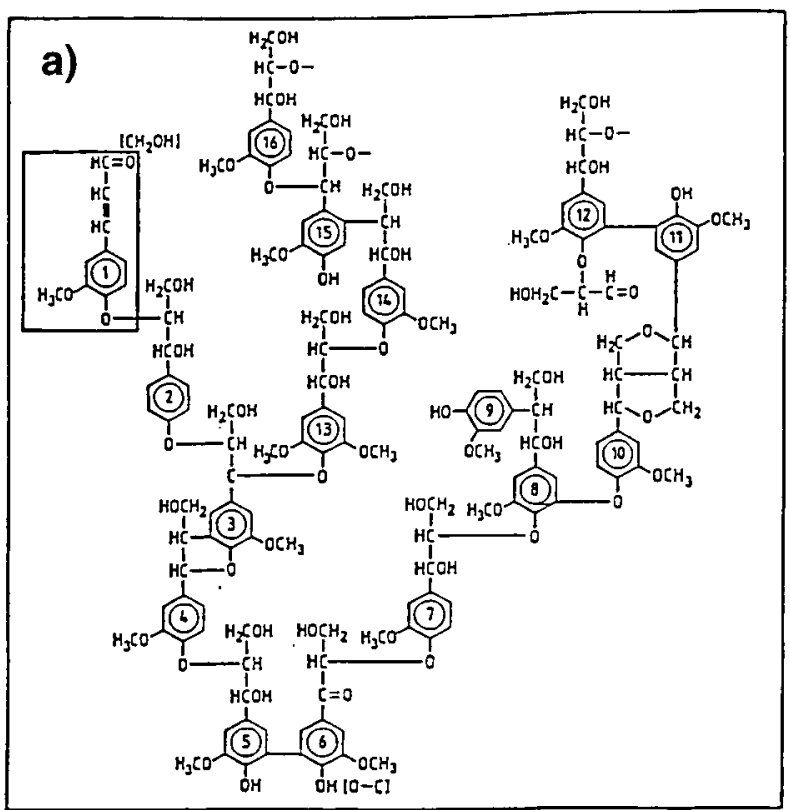

b)

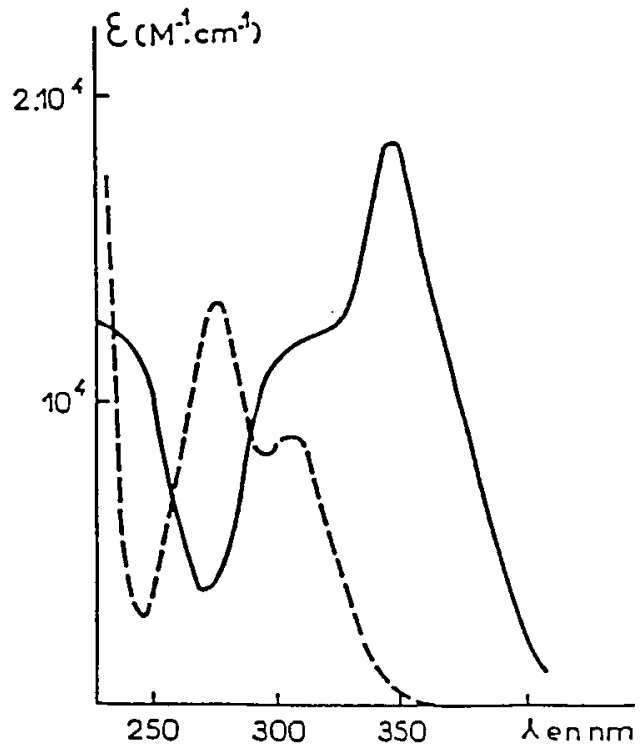

phore $\mathrm{B}$, une « queue » de bande d'absorption dans le domaine visible (figure $6 b$ ). Les échelles de temps dans lesquelles évoluent les processus photochimiques primaires permettent d'admettre qu'il n'y a pas d'effet de conjugaison entre les différents chromophores qui absorbent simultanément le rayonnement solaire. De ce fait, on peut envisager séparément les études des processus photochimiques primaires induits sur les chromophores $\mathrm{A}$ et $\mathrm{B}$. Les études fondamentales de ces processus pri- c)

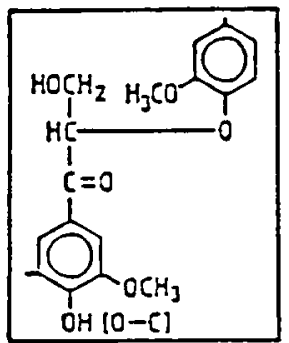

Chromophore A

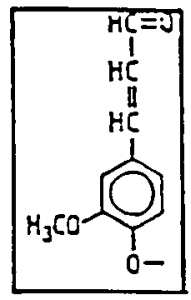

Chromophore B
Figure 6. (a) Structure des lignines de bois d'épicéa [1]. (b) Structure des chromophores A et B. (c) Spectres UV-visible de molécules modèles des chromophores A [2] et B [53].

maires, la caractérisation des espèces transitoires et des photoproduits stables ont été conduites soit sur des lignines isolées $[9,19,20,27,31,37,38]$ soit en solution à l'aide de molécules modèles des chromophores [6-8, $17,31-33,45,53,55]$. Les analyses des photoproduits stables formés lors d'une irradiation stationnaire ou des espèces transitoires en spectroscopie laser ont mis en évidence la nature radicalaire de la photodégradation à la lumière solaire des lignines. Les voies de désactivation des états triplets des chromophores A et B conduisent toutes à la formation d'un radical à structure phénoxyle (gaïacoxyle dans le cas d'une essence résineuse) à côté d'autres espèces radicalaires comme des radicaux cétyles ou phénacyles (schéma 1) ou de composés carboxyliques ou aldéhydiques non radicalaires (schéma 2). Les singulets que nous avons détectés en RPE au cours de l'irradiation des lignines en poudre ou en solution concentrée dans le dioxane ne peuvent être attribués qu'à une seule espèce radicalaire. L'allure et la position du singulet observé pour tous les types de lignines étudiés ainsi que la durée de vie des espèces radicalaires formées sont compatibles avec un radical à structure phénoxyle stabilisé par résonance. Le radical cétyle formé de façon concomitante avec le radical gaïacoxyle lors de la photoréduction de l'état triplet du chromophore A (voie 1, 
schéma 1) n'est pas détecté par spectroscopie RPE. Sa formation a pourtant été clairement mise en évidence par analyse des spectres UV-visible des espèces transitoires formées lors de la photolyse éclair des solutions de lignines de bas poids moléculaire ou de molécules modèles comme la vaniline ou le p-hydroxybenzaldéhyde $[37,38]$. Ces spectres présentent deux maxima d'absorption à 410 et $460 \mathrm{~nm}$ que les auteurs ont attribués respectivement aux radicaux phénoxyles et cétyles. Dirckx [11], en étudiant l'évolution des spectres d'absorption UV-visible d'une coupe microtomée de bois d'Abies grandis au cours d'une irradiation de type solaire, n'a pas observé d'augmentation de l'absorption à $460 \mathrm{~nm}$. Ces résultats suggèrent, qu'en irradiation continue, la concentration à l'état stationnaire en radiaux cétyles est trop faible pour être détectée par spectroscopie RPE ou par l'augmentation de l'absorption à $460 \mathrm{~nm}$.

La faible valeur de cette concentration en radicaux cétyles peut avoir deux origines :

- la désactivation de cette espèce radicalaire est trop rapide ;
- la formation du radical cétyle est concurrencée par la coupure de la liaison éther- $\beta$-aryle (voie 3, Schéma 1 ).

Les réactions de coupure à partir d'un état triplet sont souvent plus rapides que la photoréduction de cet état par arrachement d'un atome d'hydrogène sur une autre molécule qui fait intervenir un processus bimoléculaire $[14,15,34]$. Ce phénomène est certainement accentué en milieu condensé où la diffusion des espèces est très limitée.

La réaction de coupure de la liaison éther- $\beta$-aryle a été mise en évidence par spectroscopie laser d'une molécule de type $\beta-0-4 \alpha$ carbonylée [53]. Deux espèces transitoires attribuées à des états triplets de la molécule correspondant à deux conformations différentes [44] sont des intermédiaires réactifs qui se photolysent de façon efficace même en milieu dégazé [53]. En caractérisant les photoproduits formés, cet auteur confirme que l'acte primaire de la réaction est un processus intramoléculaire de scission homolytique de la liaison éther- $\beta$-aryle conduisant à la paire de radicaux gaïacoxyle et phénacy-

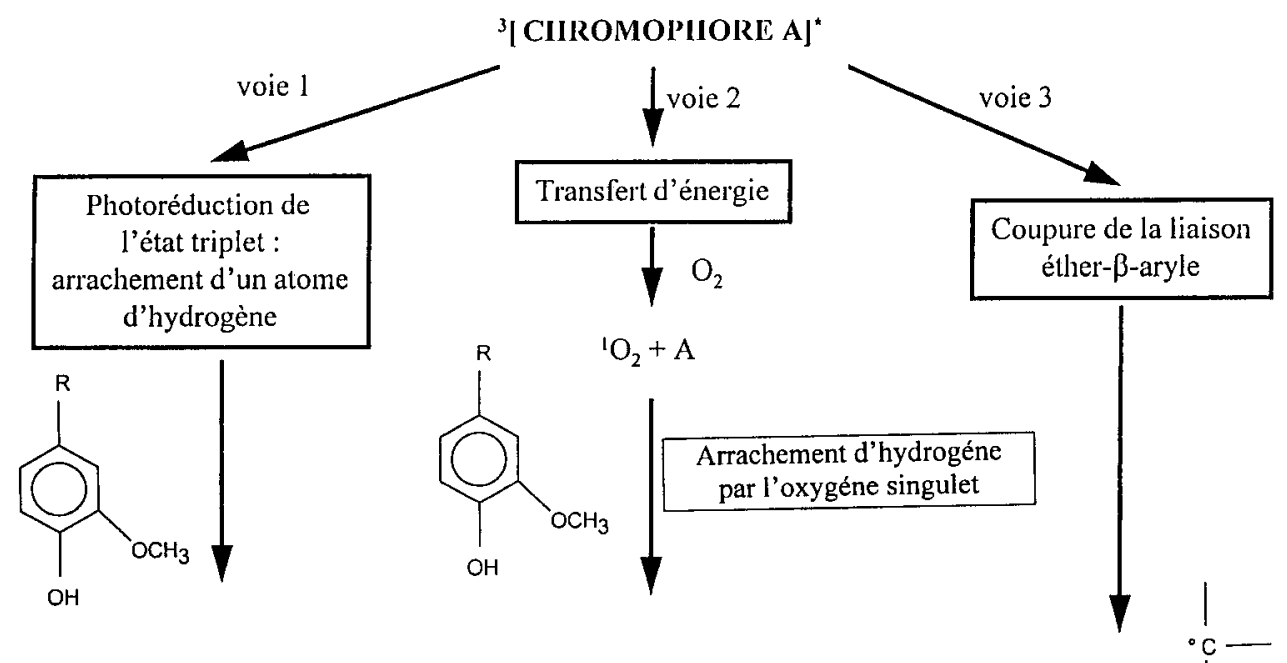

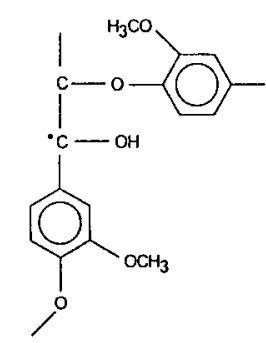

radical cétyle $\lambda \approx 460 \mathrm{~nm}$

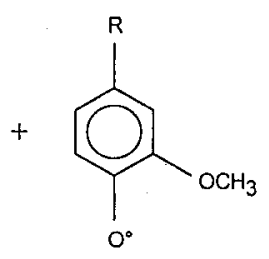

Radical gaïacoxyle $\lambda=410-430 \mathrm{~nm}$

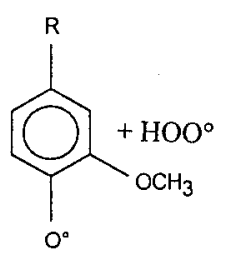<smiles>[R]c1ccc([O-])c(OC)c1</smiles>

radical phénacyle

Schéma 1. Désactivation de l'état triplet du chromophore A. 


\section{${ }^{3}\left[\right.$ CHROMOPHORE B] ${ }^{*}$}
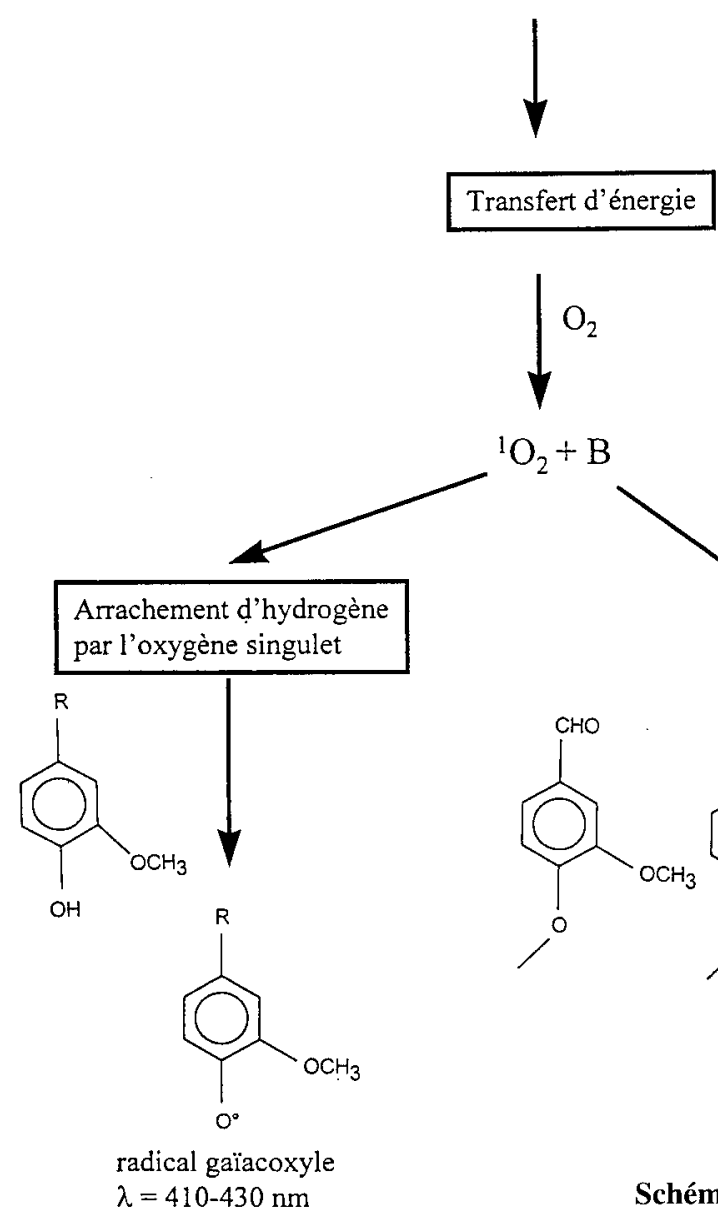

Transfert d'énergie
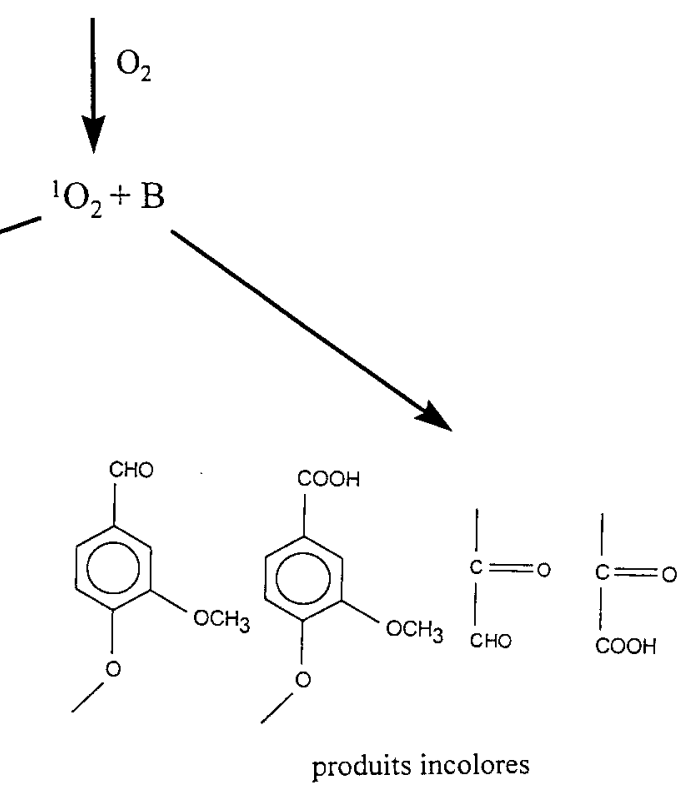

le. Cette réaction de coupure de la liaison éther- $\beta$-aryle a également été mise en évidence à partir de l'état singulet de molécules modèles de chromophores des lignines [42]. Ainsi, la réactivité des états singulets intervient dans la réaction de photodégradation des lignines et participe à la formation des radicaux gaïacoxyle et phénacyle. Comme pour le radical cétyle, la concentration à l'état stationnaire en radicaux phénacyles semble trop faible pour une détection en RPE. En présence d'oxygène, on peut envisager la désactivation des états triplets des chromophores par transfert d'énergie triplet-triplet avec formation d'oxygène singulet qui peut réagir en arrachant un atome d'hydrogène d'un groupe phénolique des lignines pour produire le radical gaiacoxyle (voie 2 schéma 1, schéma 2) $[17,18]$. Gellerstedt et Lindford [18] ont montré que pour le chromophore carbonylé A, la formation des radicaux gaïacoxyles par arrachement d'un atome d'hydrogène d'une fonction phénolique se

fait préférentiellement en solution par photoréduction de l'état triplet (voie 1, schéma 1) plutôt que par le mécanisme faisant intervenir l'oxygène singulet (voie 3 , schéma 1). D'une étude de différents modèles de lignines traitées en solution par l'oxygène singulet formé chimiquement, Nimz et Turzik $[39,40]$ concluent que l'arrachement d'un atome d'hydrogène d'une fonction phénolique par l'oxygène singulet est peu probable et proposent un mécanisme non radicalaire avec formation d'une cyclohexadiénone. Les études de la photochimie du coniféraldéhyde [17], molécule modèle des chromophores $\mathrm{B}$, ont mis en évidence que la désactivation de l'état triplet pouvait se faire par transfert d'énergie triplet-triplet avec l'oxygène. L'oxygène singulet formé peut réagir :

- en arrachant un atome d'hydrogène phénolique pour produire un radical phénoxyle; 
- sur la double liaison du coniféraldéhyde avec formation de composés non radicalaires.

Les résultats que nous avons obtenus par RPE montrent que l'oxygène a une influence sur le signal avant l'irradiation (tableau III) et sur la cinétique de formation des radicaux lors de l'irradiation des lignines brutes et purifiées en poudre. On peut penser qu'en milieu solide, la mobilité réduite rend difficile la photoréduction de l'état triplet qui nécessite l'arrachement d'un atome d'hydrogène d'un groupe phénolique d'une molécule de lignine par un carbonyle excité d'un chromophore porté par une autre molécule de lignine. En revanche, une molécule d'oxygène singulet moins encombrante présente une mobilité plus importante et peut servir de transfert d'énergie en milieu condensé. Pour la lignine acétylée, nous avons observé que l'oxygène n'avait pas d'influence sur la concentration en radicaux à l'état stationnaire en lumière ambiante (tableau II). Lors du traitement d'acétylation, les atomes d'hydrogène phénolique sont remplacés par des restes acétyles de telle sorte que les radicaux phénoxyles sont uniquement formés par la coupure de la liaison éther- $\beta$-aryle, seule voie de désactivation du triplet du chromophore carbonylé ne nécessitant pas l'arrachement d'un atome d'hydrogène labile. Cette réaction de coupure n'est pas influencée par la présence d'oxygène. Cette remarque explique également la faible intensité des signaux RPE observés avec la lignine acétylée. Castellan [9] propose la formation de radicaux phénoxyles par photolyse directe des chromophores phénoliques absorbant un rayonnement de longueur d'onde supérieure à $300 \mathrm{~nm}$. La formation de radicaux phénoxyles, en présence ou en absence d'oxygène, a été mise en évidence sur des composés phénoliques par photolyse éclair [13]. Ainsi, en absence d'oxygène, la formation des radicaux phénoxyles sur les lignines brutes et purifiées peut être envisagée par photolyse directe, par photoréduction de l'état triplet et par coupure de la liaison éther- $\beta$-aryle. Les faibles concentrations stationnaires en espèces radicalaires observées lors de l'analyse des échantillons dégazés de poudre de lignines par rapport aux échantillons aérés peuvent être la conséquence de mécanismes différents des réactions de désactivation de ces radicaux. Nous avons observé que l'oxygène accélère la diminution de l'intensité du signal RPE lors d'un stockage à l'obscurité après arrêt de l'excitation lumineuse. De nombreux travaux sur la caractérisation des photoproduits responsables du jaunissement des composés lignocellulosiques $[4,10,20,28-33]$ ont suggéré la formation par voie oxydative de structures de type quinonique à partir des radicaux phénoxyles. En absence d'oxygène, les radicaux phénoxyles se désactiveraient essentiellement par une réaction équilibrée de dimérisation [21]. Cet auteur évoque le rôle de réservoir de radicaux de ce dimère lors de l'irradiation de solu- tions dégazées de molécules modèles des lignines. Dirckx [11] a pu confirmer cet effet de réservoir en suivant les modifications des spectres UV-visible d'une coupe microtomée de bois d'Abies grandis préalablement irradiée au cours d'un stockage à l'obscurité et dans différentes atmosphères. Les observations que nous avons faites sur les signaux lors de la mise en l'air d'un échantillon de poudre de lignines ayant subi un dégazage peuvent être interprétées par une telle réaction de dimérisation équilibrée : au cours du stockage à l'obscurité après arrêt de l'excitation lumineuse, la concentration en radicaux est régie par la position de l'équilibre ; lors de la mise à l'atmosphère, nous introduisons une perturbation qui déplace l'équilibre vers la forme radicalaire ce qui provoque l'augmentation de l'intensité du signal RPE.

L'hypothèse consistant à attribuer le signal RPE observé lors de l'irradiation des lignines à des radicaux phénoxyles n'étant pas mise en défaut par les résultats expérimentaux, nous avons cherché à la confirmer. Pour cela, nous avons suivi par spectroscopie RPE la réaction de transfert radicalaire du radical $\mathrm{DPPH}^{\circ}$ (2,2 diphényl1-picrylhydrazyle) sur les lignines en solution dans le dioxane. Le radical libre $\mathrm{DPPH}^{\circ}$ ne réagit ni avec l'oxygène ni avec lui-même, par contre il disparaît rapidement en présence de substances possédant des atomes d'hydrogènes mobiles [43]. Ainsi, les atomes d'hydrogène des fonctions phénoliques des lignines sont suffisamment labiles pour réagir sur le $\mathrm{DPPH}^{\circ}$ et former des radicaux phénoxyles:
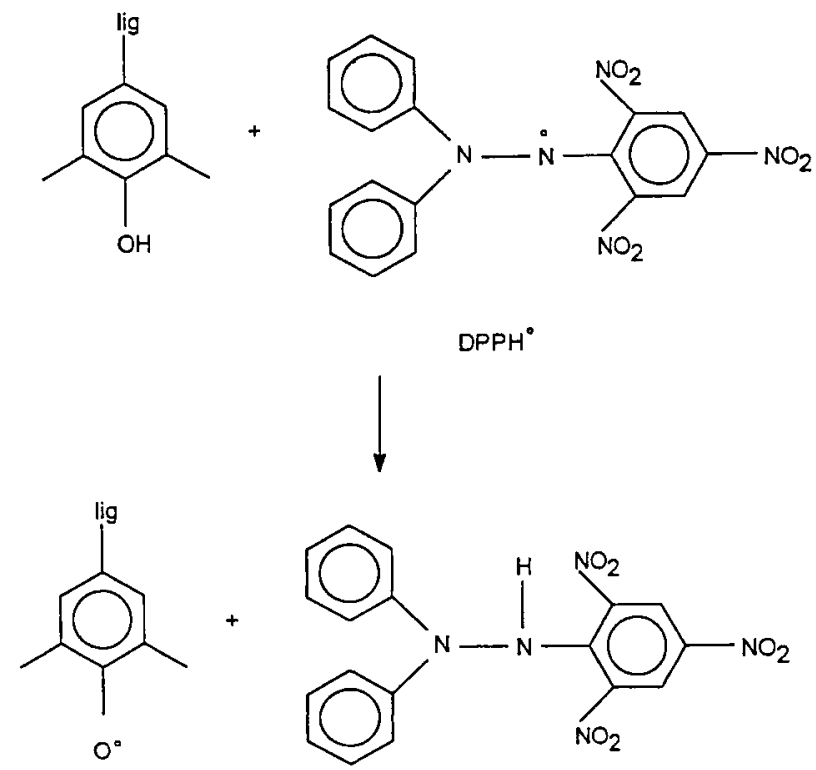

radical phénoxyle 
Tableau VI. Calcul de la concentration initiale relative en chromophores à partir de la modélisation.

\begin{tabular}{lccc}
\hline Lignine : & $\mathrm{K}$ & $\mathrm{k}_{\mathrm{l}} / \mathrm{k}_{2}-\mathrm{k}_{1}$ & $\begin{array}{c}\text { concentration initiale } \\
\text { relative en chromophores }\end{array}$ \\
\hline brute & 6,390 & $6,22 \cdot 10^{-3}$ & 1 \\
purifiée & 9,766 & $6,42 \cdot 10^{-3}$ & 1,48 \\
acétylée & 4,840 & $6,05 \cdot 10^{-2}$ & 0,08 \\
\hline
\end{tabular}

Tableau VII. Comparaison des cinétiques de desactivation des espèces radicalaires en phase solide et en solution.

\begin{tabular}{lccc}
\hline Lignine : & $\begin{array}{c}\tau^{\prime} \text { en phase solide } \\
\text { en min }\end{array}$ & $\begin{array}{c}\tau^{\prime} \text { en phase liquide } \\
\text { en min }\end{array}$ & $\begin{array}{c}\tau^{\prime} \text { liquide/ } \\
\tau^{\prime} \text { solide }\end{array}$ \\
\hline brute & 3,63 & 33 & 9,1 \\
purifiée & 3,29 & 41 & 12,7 \\
\hline
\end{tabular}

Le signal RPE du radical DPPH ${ }^{\circ}$ en solution dans le dioxane (figure 7a) disparaît très rapidement lorsqu'on ajoute des lignines pour donner place à un singulet (figu$r e 7 c$ ) dont la position et la largeur sont tout à fait comparables à celui obtenu par l'irradiation des lignines. La cinétique de disparition du radical $\mathrm{DPPH}^{\circ}$ en présence de lignines peut être suivie en mesurant au cours du temps l'intensité du signal RPE en fixant le champ magnétique à la valeur correspondant à l'absorption de la microonde par le radical $\mathrm{DPPH}^{\circ}$ (figure 7) : cas de la lignine purifiée). Une telle méthodologie par spectroscopie RPE a déjà été appliquée pour suivre la cinétique de la réaction de transfert du $\mathrm{DPPH}^{0}$ sur des molécules phénoliques synthétiques [56] ou naturelles [41,43]. Dans le cas des lignines, il est très difficile d'accéder à la constante de vitesse de cette réaction de transfert par une modélisation des résultats expérimentaux ; pour cela, il faudrait pouvoir se placer dans des conditions de pseudo-premier ordre (concentration de l'un des réactifs en excès). La forte masse moléculaire des lignines et leur solubilité limitée dans le dioxane ne permettent pas d'obtenir un excès en fonctions phénoliques portées par les lignines. De plus, aux fortes concentrations en DPPH ${ }^{\circ}$, l'absorption de la microonde par ce radical est pratiquement totale et la mesure RPE devient impossible. La figure 8 montre que, dans nos conditions expérimentales, il faut environ 10 min pour obtenir la moitié de la concentration initale en $\mathrm{DPPH}^{0}$. Cette réaction de transfert radicalaire est responsable de la disparition de la coloration violette d'une solution de $\mathrm{DPPH}^{0}$ dans le dioxane lorsqu'on ajoute des lignines, pour donner une coloration jaunâtre (attribuée aux radicaux phénoxyles $\left(\lambda_{\max } \sim 430 \mathrm{~nm}\right)$ et aux produits formés par désactivation de ces mêmes radicaux). Le composé $\mathrm{DPPH}_{2}$ est incolore. Cette variation de couleur a permis de quantifier la labilité des atomes d'hydrogène phénolique de molécules modèles des lignines et des substances extractibles du bois en mesurant par spectrophotométrie à écoulement bloqué, dans le domaine visible, la constante de vitesse de la réaction de transfert radicalaire sur le $\operatorname{DPPH}^{0}[22,35]$.
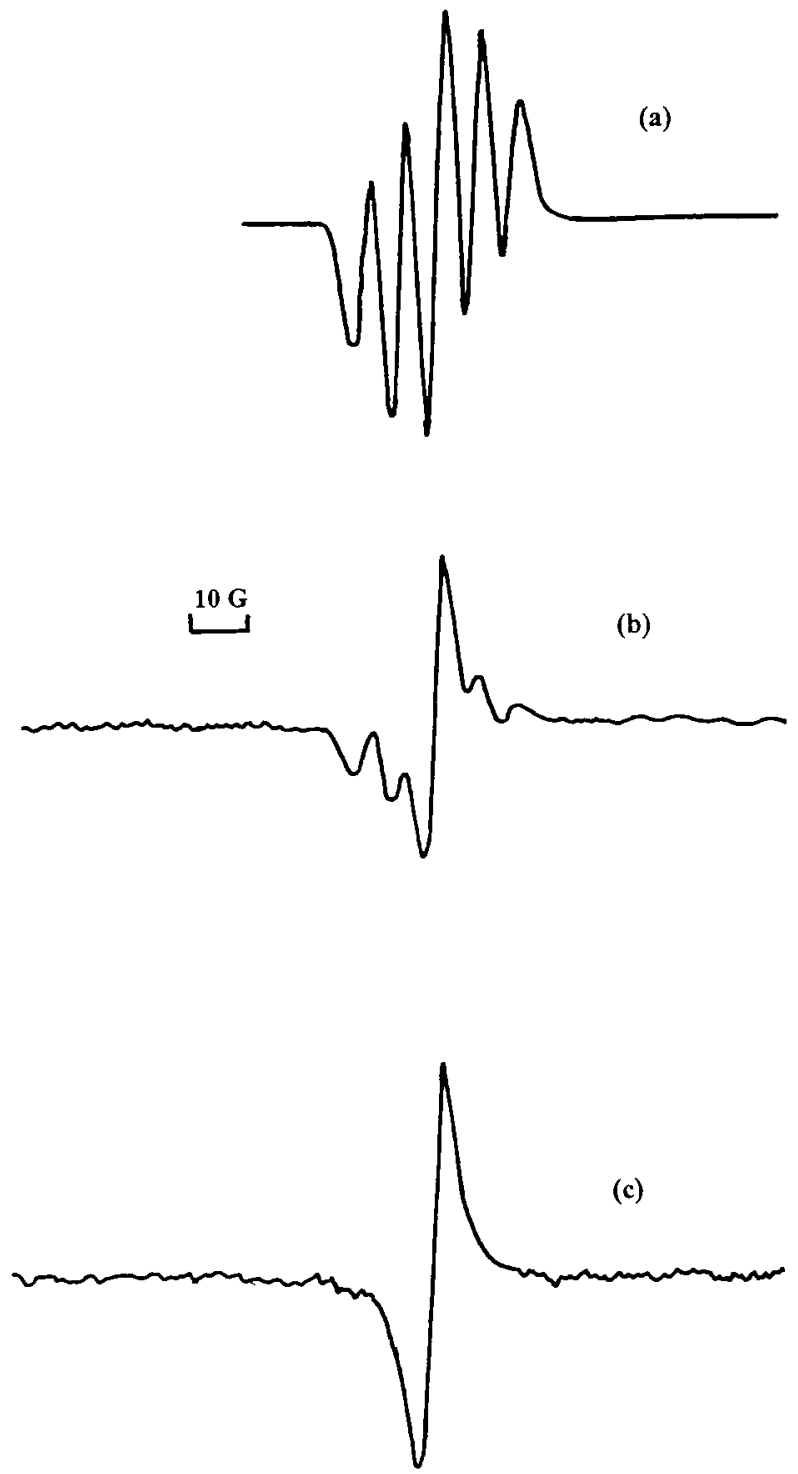

Figure 7. Suivi de la réaction de transfert radicalaire du $\mathrm{DPPH}^{\circ}$ sur les lignines.

(a) $\mathrm{DPPH}^{\circ}$ en solution dans le dioxane.

(b) $\mathrm{DPPH}^{\circ}+$ liginines en défaut.

(c) $\mathrm{DPPH}^{\circ}+$ lignines en excès. 


\section{Conclusion}

Cette étude met en évidence le rôle des radicaux phénoxyles dans les réactions de photodégradation des lignines. L'analyse de la lignine acétylée montre que ce traitement, en diminuant la concentration en groupes hydroxyphénoliques libres, freine la formation des radicaux phénoxyles. Il a été observé que la modification chimique du bois ou des pâtes à papier mécaniques par estérification ou éthérification des groupes phénoliques $[3,5,16,29,46-48]$ permettait une stabilisation de la couleur lors d'une exposition à un rayonnement de type solaire. Kringstadt et Lin [27] avaient également mis en évidence que le blocage chimique des fonctions hydroxyphénoliques n'est pas suffisant pour stabiliser totalement la couleur des composés lignocellulosiques. Nos observations par RPE confirme que la formation des radicaux phénoxyles n'est pas totalement inhibée par le
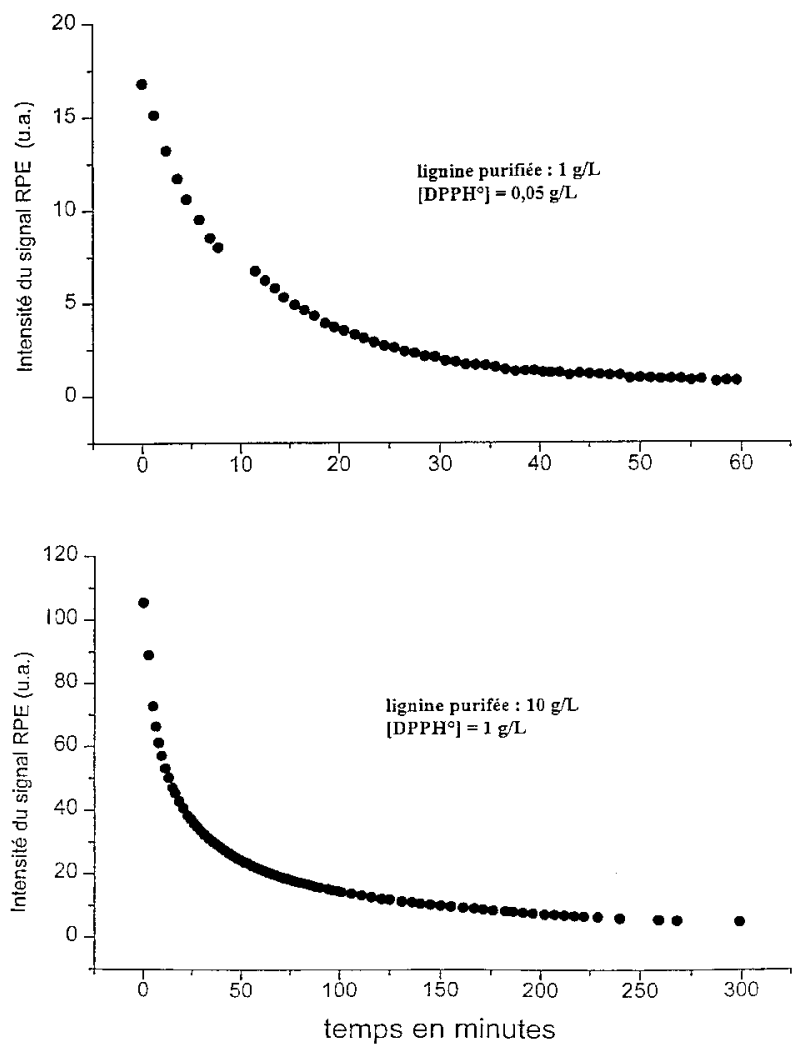

Figure 8. Suivi de la désactivation du radical $\mathrm{DPPH}^{\circ}$ en présence de lignines purifiées en solution dans le dioxane. traitement d'acétylation ; les réactions de coupure de la liaison éther- $\beta$-aryle permettant la production de ces radicaux sans intervention des hydrogènes labiles des fonctions phénoliques. Ainsi un traitement combinant l'estérification des fonctions phénoliques et la réduction des groupes carbonyles par l'action de borohydrure de sodium assure une meilleure stabilisation de la couleur des composés lignocellulosiques $[5,16,24,50]$. La capacité des lignines à produire des radicaux phénoxyles par transfert radicalaire confère à ce composé un rôle d'antioxydant. D'une manière générale, l'antioxydation consiste à remplacer dans le milieu, des radicaux oxygénés très réactifs (le plus souvent $\mathrm{ROO}^{\circ}$ ) par des radicaux stables et ainsi bloquer les réactions en chaînes de dégradation oxydantes schéma 3). Dans le cas des lignines, le radical phénoxyle est également capable de désactiver les radicaux oxygénés par une réaction de déméthylation $[24,49]$.<smiles>CCP(=O)(O)C1(OC)C=C([Al])C=C(C)C1=O</smiles>

Toutefois, en phase solide, ces réactions bimoléculaires de transfert sont défavorisées car elles nécessitent une mobilité des espèces réagissantes. L'étude par RPE des solutions de lignines soumises à l'irradiation a montré que les cinétiques de formation des espèces radicalaires étaient différentes de la phase solide et la concentration stationnaire en radicaux semblait résulter de réactions consécutives. Cette observation met en évidence que les extrapolations des résultats obtenus en solution à un matériau lignocellulosique en phase solide doivent être prises avec précaution.

Dans le bois, il a été montré que les lignines comme les substances extractibles avaient un rôle « protecteur » de la cellulose vis-à-vis du rayonnement solaire. À l'état isolé, la cellulose exposée à un rayonnement solaire subit une dégradation qui se manifeste par une perte de masse et une diminution du degré de polymérisation [26]. Dans un matériau lignocellulosique, la photochimie de la cellulose est totalement modifiée par la présence des lignines. En utilisant la spectroscopie RPE, Hon [25] a montré que les espèces radicalaires formées par irradiation de la cellulose isolée ne sont plus détectées en présence des lignines. Cet auteur en conclut que les lignines sont capables de désactiver l'état excité de la cellulose 
par un processus de transfert d'énergie dans lequel les lignines joueraient le rôle d'accepteur:

$$
\text { cellulose } \stackrel{h v}{\longrightarrow}{ }^{3}[\text { cellulose }]^{*} \stackrel{\text { lignines }}{\longrightarrow}{ }^{3}[\text { lignines }]^{*}
$$

En phase solide, ce mécanisme de transfert d'énergie est favorisé par rapport à des réactions de transfert radicalaire sur les radicaux formés par photolyse de la cellulose.

La protection de la cellulose résulterait donc d'une triple action des lignines :

- rôle d'absorbeur des photons UV-visible par sa coloration ;

- rôle de sensibilisateur en désactivant les états excités de la cellulose ;

- rôle d'antioxydant par formation des radicaux phénoxyles.

En plus de l'aspect cognitif sur la photodégradation et donc sur la durabilité naturelle du matériau bois, cette étude des propriétés antioxydantes ouvre des perspectives intéressantes de valorisation des lignines, sous-produits de l'industrie papetière. Il peut être envisagé de substituer des antioxydants phénoliques synthétiques utilisés en agroalimentaire, en cosmétologie, comme additif de stabilisation des polymères par des composés phénoliques naturels comme les lignines ou les substances extractibles du bois.
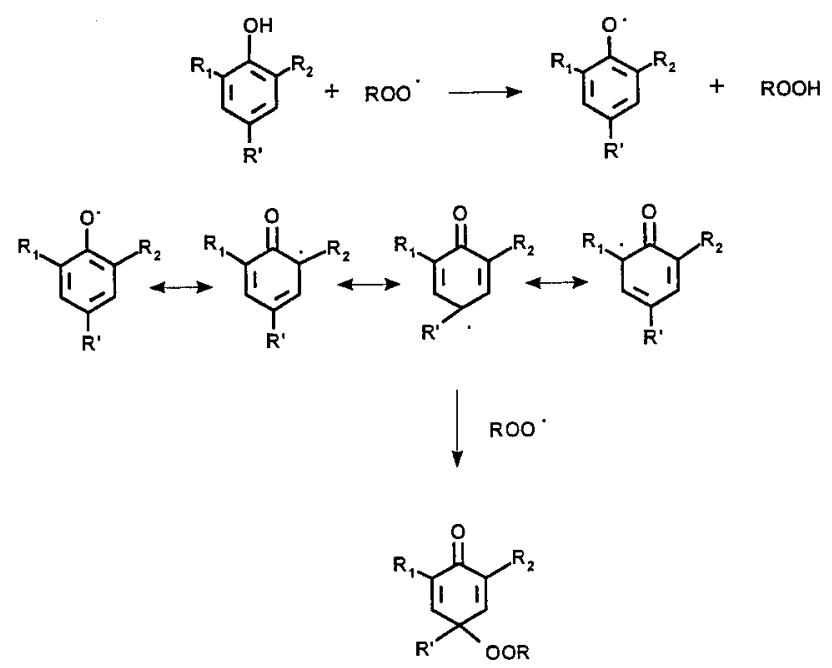

Schéma 3. Mécanisme de fonctionnement des antioxydants phénoliques.
Remerciements : Les auteurs remercient EcosConicyt et le Fondecyt (Projet $n^{\circ} 195089$ ) qui ont soutenu financièrement ce travail.

\section{Références}

[1] Adler E., Lignin chemistry: past, present and future, Wood Sci. Technol. 11 (1977) 169-213.

[2] Adler E., Marton J., Zur Kenntnis der Carbonylgruppen in lignin, Acta Chem. Scand. 13 (1959) 75-96.

[3] Andrew D.H., Singh R.P., Bleaching Groundwood Pulp, Canadian Patent 810, 571, 15 April, 1966.

[4] Argyropoulos D.S., Heitner C., Morin G., Holzforschung 46 (1992) 211-215.

[5] Cameron P.A., Pizzi A., Holz Roh Werkstoff 43 (1969) 149-151.

[6] Castellan A., Vanucci C., Desvergne J.P., BouasLaurent H., Hauteville M., Chadenson M., Joussot-Dubien J., Etude photochimique de dimères modèles des lignines de type $\alpha-0-4$ et $\beta-0-4$ méthyles, C.R. Acad. Sci. Paris 301 (II) (1985) 21-26.

[7] Castellan A., Colombo N., Nourmamode A., BouasLaurent H., Forniet de Violet P., Photodegradation of lignin: a photochemical study of bleached CTMP and lignin model molecules, Tappi Proc. Wood Pulping Chem. 1 (1989) $421-430$.

[8] Castellan A., Colombo N., Cucuphat C., Forniet de Violet P., Photodegration of lingin: a photochemical study of phenolic and carbonyl $3-0-4$ lignin model dimer 4-hydroxy-3methoxy - $\alpha\left(2^{\prime}\right.$ methoxy phenoxy) acetophenone, Holzforschung 43 (1989) 179-185.

[9] Castellan A., Colombo N., Nourmamode A., Zhu J.H., Lachenal D., Davidson R.A., Dunn L., Discoloration of $\alpha$-carbonyl free lignin model compounds under UV light exposure, J. Wood Chem. Technol. 10 (4) (1990) 461 - 477.

[10] Castellan A., Nourmmamode A., Jaeger C., Forsskahl I., Photochemistry of quinons and hydroquinons in solid 2hydroxypropylcellulose films and on filter paper: UV-visible absorption and diffuse reflectance spectroscopy, in : Heitner C., Scaiano J.C., (Ed.), Photochemistry of Lignocellulosic Materials, ACS Symposium Series 531, Washington, 1993, pp. $60-76$.

[11] Dirckx O., Etude du comportement photochimique de l'Abiès Grandis sous irradiation solaire, Doctorat de $3^{\circ} \mathrm{Cycle}$ en Sciences du Bois, Université Henri Poincaré, Nancy 1, 1988.

[12] Dirckx O., Triboulot M.C., Merlin A., Deglise X., Modification de la couleur du bois d'Abies grandis exposé à la lumière solaire, Ann. Sci. For. 49 (1992) 425-447.

[13] Ebbesen T.N., Previtali C.M., Spectral evidence for long-lived radical electron pair: photoionization of phenol in alcohols, Photochemistry 31 (1985) 193-194.

[14] Fouassier J.P., Merlin A., Photochemical $\alpha$-cleavage of hydrogen abstraction in deoxybenzoïn: a laser spectroscopy investigations, Can. J. Chem. 57 (1979), 2812- 2818. 
[15] Fouassier J.P., Merlin A., Laser investigation of Norrish type 1 photoscission in the photo initiator Irgacure 2-2 diméthoxy-2-phenyl acetophenone, J. Photochem. 12 (1980) 17-23.

[16] Francis R.C., Dence C.W., Alexander T.C., Agnemo R., Moris S., Photostabilization of thermomechanical pulps by alkylation and borohydride reduction, Tappi J. 74/12 (1991) 127-134.

[17] Gellerstedt G., Petterssson E.L., Light induced oxidation of lignin: The behaviour of structural units containing a ring conjugated double bond, Acta Chem. Scand. B29 (1975) $1005-1010$.

[18] Gellerstedt G., Lindfors A., Canadian Wood Chemistry Symposium, Mont-Gabriel, 1976, pp. 21-24.

[19] Gellerstedt G., Petterssson E.L., Light induced oxidation of lignin. Part 2. The oxidative degradation of aromatic sings, Swensk paperation 80 (1977) $15-21$.

[20] Gellerstedt G., Petterssson E.L., Autoxidation of lignin, Swensk paperation 83 (1980) 314-318.

[21] Girard P., Chimie et photochimie de modèles de lignine monomères, Thèse de Doctorat de l'Université de Bordeaux 2, 1984.

[22] Harroudi Z., Photodégradation et pouvoir antioxydant des substances extractibles du bois. Tanins flavanoïdes, Thèse de Doctorat d'état, Université de Fès (Maroc), 1997.

[23] Harroudi Z., Merlin A., La chimie radicalaire et le matériau bois : la spectoscopie de résonance paramagnétique électronique (RPE ou ESR), les Cahiers Scientifiques du Bois Instrumentation 1 (1997) 17 - 32.

[24] Heitner C., Light-induced yellowing of wood-containing papers: An evolution of the mechanism, in : Heitner C., Scaiano J.C. (Ed.), Photochemistry of Lignocellulosic Materials, ACS Symposium Series 531, Washington, 1993, pp. $2-25$.

[25] Hon D.N.S., Formation of free radicals in photo irradiated cellulose, J. Polym. Sci. Chem. Ed. 13 (1975) 2641-2652.

[26] Hon D.N.S., Photooxidative degradation of cellulose: Reaction of the cellulosic free radicals with oxygen, J. Polym. Sci. Chem. Ed. 17 (1979) 441-454.

[27] Kringstadt K.P., Lin S.Y., Mechanism in the yellowing of hight yield pulp by light: Structure and reactivity on free radical intermediates in the photodegradation of lignin, Tappi $\mathbf{J}$. 53 (12) (1970) $2296-2301$.

[28] Leary G.J., The yellowing of wood by light, Tappi J. 51 (6) (1968) 257-260.

[29] Leary G.J., Photochemical production of quinoid structures in wood, Nature 217 (1968) 672-673.

[30] Lebo S.E., Lonsky W.F.W., Mc Donough T.J., Medveca P.J., Dimmel D.R., The occurrence of light-induced formation of ortho-quinonoid lignin structures in white spruce refimer mechanical pulp, J. Pulp Paper Sci. 16 (1990) J 139.

[31] Lin S.Y., Kringstadt K.P., Photosensitive group in lignin and lignin model compounds, Tappi J. 53 (4) (1970) $658-663$.
[32] Lin S.Y., Kringstadt K.P., Stabilization of lignin and lignin model compounds photodegradation, Tappi J. 53 (9) (1970) 1675-1677.

[33] Lin S.Y., Kringstadt K.P., Some reaction in the photoinduced discoloration of lignin, Norsk. Shogindustrie 25 (9) (1977) 252-256.

[34] Lougnot D.J., Merlin A., Salvin R., Fouassier J.P., Faure J., Time resolved spectroscopy of carbonyl compounds, Nuovo Ciments 63 (1981) 218-225.

[35] Martin F., Influence des substances extractibles sur le comportement photochimique du bois de chêne : propriétés antioxydantes de ces composés, Thèse de Doctorat de l'Université Henri Poincaré Nancy 1, 1996.

[36] Mazet J.F., Triboulot M.C., Janin G., Merlin A., Deglise X., Modification de la couleur du bois de chêne européen exposé à la lumière solaire, Ann. Sci. For. 50 (1993) 119-146.

[37] Neumann M.G., de Groote R.A., Machado A.E., Flash photolysis of Ignin. Part I: Deaerated solutions of dioxanelignin, Polymer Photochem. 7 (1986) 401- 407.

[38] Neumann M.G., DE Groote R.A., Machado A.E., Flash photolysis of lignin. Part II: Oxidative photodegradation of dioxane-lignin, Polymer Photochem. 7 (1986) 46I-468.

[39] NimZ H., Turzik G., Investigation of the reactivity of lignin to wards oxygen an peroxides, Kemia Kemi 9 (1980) $477-480$.

[40] NimZ H., Turzik G., Reaction of lignin with singulet oxygen. Part 1: oxidation of monomeric and dimeric model compounds with sodium hypochlorite hydrogen peroxide, Cell. Chem. Technol. 14 (1980) 727-742.

[41] Noferi M., Masson E., Merlin A., Pizzi A., Deglise X., Antioxydant characteristics of hydrolysable and polyflavanoid tannins: an ESR kinetics study, J. Appl. Polym. Sci. (1997) $475-482$.

[42] Palm W.V., Dreeskamp H., Bouas -Laurent H., Castellan A., The photochemistry of $\alpha$-phenoxyacetaphenones. Investigated by flash CIDNP Spectroscopy, Ber. Bunsengues Phys. Chem. 96 (1) (1992) 50-61.

[43] Rousseau-Richard C., Inhibition de l'oxydation induite de linoléate de méthyle par des dérivés phénoliques ou par les vitamines $\mathrm{E}$ et $\mathrm{C}$, Thèse de Doctorat d'état de l'université Henri Poincaré Nancy 1, 1986.

[44] Scaiano J.C., Netto-Ferreira J.C., Photochemistry of $\alpha$ phenoxy acetophenone: An interesting case of intermolecular triplet desactivation, J. Photochem. 32 (1986) 253-259.

[45] Schmidt J.A., Goldszmidt E., Heitner C., Scaiano J.C., Berinstain A.B., Johnston L.J., Photodegradation of Guaiacoxyacetoveratrone: Triplet-State reactivity induced by protic solvents in : Heitner C., Scaiano J.C. (Ed.), Photochemistry of Lignocellulosic Materials, ACS Symposium Series 531, Washington, 1993, pp. 122-128.

[46] Schmidt J.A., Heitner C., Light-induced yellowing of mechanical and ultra-high yield pulps. Part I: Effect of methylation $\mathrm{NaBH}_{4}$ reduction and ascorbic acid on chromophore formation, J. Wood Chem. Technol. 11 (4) (1991) 397-406. 
[47] Schmidt J.A., Heitner C., Light-induced yellowing of mechanical and ultra-hight yield pulps. Part II: The role of etherified guaiacylglycerol- $\beta$-arylether groups, Proceedings of the Second European Workshop on lignocellulosics and pulp, Grenoble, 1992.

[48] Singh R.P., Bleaching groundwood pulp: Investigation of methods based on chemical modification of pulp, Tappi $\mathbf{J}$. 49 (7) (1966) 281-286.

[49] Shkrob I.A., Depen M.C., Wan J.K.S., Free radical induced oxidation of alkoxyphenols: Some insights into the process of photoyellowing of papers, Res. Chem. Intermed. 17 (1992) 271-276.

[50] Tschirner U., Dence C.N, Attemps to photostabilize norway spruce TMP by chemical modification, Paperi ja Puu 36 (4) (1988) 338-342.

[51] Urizar S., Faix O., Freer J., Baeza J., Caracterizacion de ligninas organosolv de pinus radiata y de eucalyptus globulus, Simposio chileno de quimica y fisico quimica de polimeros, Quilpue (Chili), 1992.

[52] Urizar S., Freer J., Baeza J., Deslignification de madera de pinus radiata con acido formico-acetona, IV Congress National de postgrado en quimica, Concepcion (Chili), 1992.

[53] Vanucci C., Etude photochimique de dimères modèles de la lignine en solution et à l'état adsorbé, Thèse de Doctorat de l'Université de Bordeaux 1, 1987.

[54] Vanucci C., Forniet de Violet P., Bouas-Laurent H., Castellan A., Photodegradation of lignin: a photophysical and photochemical study of a non-phenolic $\alpha$-carbonyl -0-4 lignin model dimer: 3-4 dimethoxy--(2'methoxyphenoxy acetophenone), J. Photochem. Photobiol. A: Chem. 41 (1988) 251-265.

[55] Wan J.K.S., Shkrob I.A., Depen C., Primary photophysical and photochemical processes of $\alpha$-guaiacoxyacetoveratrone in : Heitner C., Scaiano J.C. (Ed.) Photochemistry of Lignocellulosic Materials, ACS Symposium Series 531, Washington, 1993, pp. 9-110.

[56] Westfahl J., Carman C.J., Layer R.W., ESR study of phenolic antioxydants. Correlation of organic free radical stability with antioxidant activity, Rubber Chem. Technol. (1971) $402-422$.

\section{Annexe 1}

$$
\begin{gathered}
\mathrm{Si} \text { nous supposons le modèle cinétique : } \\
A \underset{k_{1}}{\longrightarrow} B^{0} \underset{k_{2}}{\longrightarrow} C \text { où } \mathrm{B}^{\circ} \text { est la seule espèce radica- }
\end{gathered}
$$

laire donnant une réponse en spectroscopie RPE, l'évolution temporelle du système est décrite par le système différentiel suivant :

$$
\begin{gathered}
\frac{d[A]}{d t}=-k_{1}[A] \\
\frac{d\left[B^{\circ}\right]}{d t}=k_{1}[A]-k_{2}\left[B^{\circ}\right] \\
\frac{d[C]}{d t}=k_{2}\left[B^{\circ}\right]
\end{gathered}
$$

De l'équation (1), on obtient : $[A]=[A]_{0} \exp \left(-k_{1} t\right)$

Dans le cas d'une réaction photochimique, la concentration $[A]_{0}$ doit correspondre à la concentration initiale en chromophores susceptibles, en absorbant le rayonnement, de produire les espèces radicalaires $\mathrm{B}^{\circ}$.

L'intégration de l'équation différentielle (2) permet d'exprimer la concentration en espèces radicalaires $\left[\mathrm{B}^{\circ}\right]$ au temps $\mathrm{t}$ d'irradiation :

$$
\left[B^{\circ}\right]=\exp \left(-k_{2} t\right)\left[\left(k_{1} / k_{2}-k_{1}\right)[A]_{0} \exp \left(k_{2}-k_{1}\right) t+\beta\right](4)
$$

$\beta$ étant une constante d'intégration.

soit

$$
\begin{gathered}
{\left[B^{\circ}\right]=\left[\left(k_{1} / k_{1}+k_{2}\right)[A]_{0} \exp \left(-k_{1} t\right)\right.} \\
\left.+\beta \exp \left(-k_{2} t\right)\right]
\end{gathered}
$$

L'intensité du signal RPE au temps $t$, I(t) est directement proportionnelle à la concentration $\left[\mathrm{B}^{\circ}\right]$ donc :

$$
I(t)=K \exp \left(-k_{1} t\right)+C \exp \left(-k_{2} t\right)
$$

Au temps $t=0, I(t)=I_{0}$ intensité du signal RPE avant l'irradiation.

D'après l'équation (6) : $\mathrm{I}_{0}=\mathrm{K}+\mathrm{C}$. On obtient alors :

$$
I(t)=K\left[\exp \left(-k_{1} t\right)-\exp \left(-k_{2} t\right)\right]+I_{0} \exp \left(-k_{2} t\right)
$$

expression qui correspond à la modélisation proposée.

La comparaison des expressions (5) et (6) montre que $\mathrm{K}$ est directement proportionnel à $\left(k_{1} / k_{2}-k_{1}\right)[A]_{0}$. 